\title{
A Mechanism to Make Authorization Decisions in Open Distributed Environments Without Complete Policy Information
}

\author{
Chiu-Man Yu and Kam-Wing Ng \\ Department of Computer Science and Engineering, \\ The Chinese University of Hong Kong \\ $\{$ cmyu, kwng\}@cse.cuhk.edu.hk
}

\begin{abstract}
To enable an open Grid environment to support organized resource sharing between multiple heterogeneous Virtual Organizations (VOs), we need to tackle the challenges of dynamic membership of VOs and trust relationships between the VOs. We propose a Dynamic Policy Management Framework (DPMF), a Conflict Analysis with Partial Information (CAPI) mechanism, and a heterogeneous authorization policy management mechanism to resolve the problems. DPMF groups VOs deploying the same model of authorization systems together to form a virtual cluster. Policy management is divided into inter-cluster heterogeneous policy management, and intra-cluster homogeneous policy management. In an open Grid environment, some VOs may prefer to keep their policy information private. The Conflict Analysis with Partial Information (CAPI) mechanism is developed to provide an approach of policy conflict analysis in open environments without complete policy information. The basis of CAPI is to generate substitution policies to replace the unknown policy information.
\end{abstract}

\section{Introduction}

Traditional security policy frameworks [1] 2] [4] deal with security policy management inside a VO. There is still little research on policy management for multiple heterogeneous VOs. We have proposed an authorization policy management framework, Dynamic Policy Management Framework (DPMF) [6], to handle this problem. DPMF is a hierarchical framework which aims to support dynamic, distributive, and heterogeneous authorization policy management for Grid environments of multiple VOs. Each VO consists of a number of nodes which can be service providers, or service requesters. Each VO has a policy server (or an agent which can access the policy repository of the VO). The policy server is a PDP (Policy Decision Point). The service providers and service requesters on the VOs are PEPs (Policy Enforcement Points) 3].

Figure 1 illustrates the DPMF's hierarchical architecture. There are three kinds of agents in the framework: Policy Agents (PA), Policy Processing Units (PPU), and Principal Policy Processing Unit (P-PPU). In DPMF, each VO 


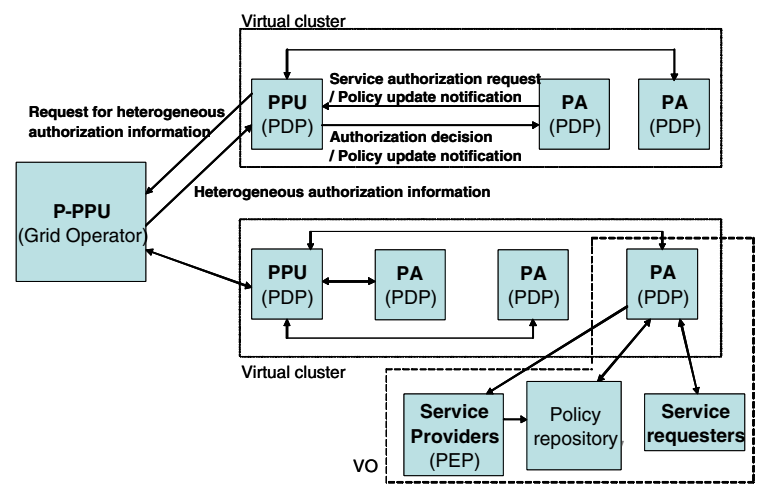

Fig. 1. DPMF architecture

needs to have a PA. The Grid Operator has a P-PPU. The DPMF groups VOs of same model of authorization system (in terms of access control model and policy model) to form a virtual cluster. Inside a virtual cluster, the workloads of policy management can be distributed among the VOs according to their trust relationships. The Conflict Analysis with Partial Information (CAPI) mechanism is developed to provide an approach to make authorization decisions in open environments without complete policy information. Section 2 and Section 3 will present the CAPI mechanism and its performance resulting from simulation.

\section{Conflict Analysis with Partial Information (CAPI)}

In an open environment, some VOs may prefer to keep their policy information private. In DPMF, a trust relationship between VOs (representing by PAs) means the willingness of disclosing their authorization policies. Since a trust relationship is not compulsory in DPMF, some PAs may not be trusted by some other PAs. During the manipulation of service authorization requests, if some of the involved PAs do not trust the PPU, the PPU is not able to retrieve certain policy information from the PAs. For example, a user from a VO plans to perform a task which involves inter-operation with service providers on several other VOs. So the user requests the PPU for authorization for the task. If some of the target service providers' PAs trust the PPU but some do not, then the PPU cannot retrieve the policy information from the untrustful PA(s). The PPU can only perform policy conflict analysis and make authorization decision with policy information from the trustful PAs. To handle the problem, we have developed a conflict analysis mechanism which requires only partial policy information. It is called "Conflict Analysis with Partial Information" (CAPI).

The main idea of our current approach of CAPI is that the PPU performs a conflict analysis with the known authorization policies, generates substitutions for unknown policies, finds out a set of conditions which can cause conflicts, then sends the set of conditions to the untrustful VO for its checking. 
A policy template consists of a Condition Set, an Action Set, a Target Identity, an Extension Set, an Evaluation Element Set, and a corresponding Priority Set. The Condition Set, Action Set, Target Identity and Extension Set are learnt from policy information provided by trustful PAs. The Evaluation Element Set stores evaluation elements which are attributes of the policy owner. The attributes are defined by the PPU. They probably include the type of service providers, the type of Virtual Organizations, security levels, etc. The Priority Set stores the weights of importance of the evaluation elements.

$(\text { Policy })_{A} \rightarrow \leftarrow(\text { Policy })_{B}$

if $(\text { Condition })_{A} \cap(\text { Condition })_{B} \neq \varnothing$

and $(\text { Action })_{A}$ is: [Permit] to access resource for (Target Identity) and $(\text { Action })_{B}$ is: [Deny] to access resource for (Target Identity)

The above expression shows a generic conflict model for policy conflict between two policies. The symbol $\rightarrow \leftarrow$ represents the conflict relationship, which is symmetric. The expression states that $(\text { Policy })_{A}$ and $(\text { Policy })_{B}$ conflict if they have an intersection of conditions which results in opposite authorization action on the resource (target services) for the Target Identity (service requester).

During conflict detection in DPMF, the PPU (or delegated PA) also needs to conclude permission conditions. An authoirzation is granted if only if there exists a permission condition set for the task in the authorization request. The permission conditions will be listed in an authorization token if the authorization for the request is positive. The method of concluding permission conditions is to intersect (i.e., AND operation) the conditions of the policies of the target services, where the action of the policies is to permit the service authorization requester to access the services. Therefore, the concluded conditions are the largest condition set which allows the requester to access the multiple target services simultaneously. The requester can then perform its task by making an access to the target services under the conditions.

\subsection{Conflict Analysis with Partial Information (CAPI) Mechanism}

The CAPI mechanism is divided into three phases. The Pre-detection phase is to prepare the "policy template database". The Detection phase is to generate substitution policies to perform conflict detection and to conclude permission conditions. The Post-detection phase is to communicate with untrustful hosts to check the conflict detection results, and finally to make authorization decisions.

In the Pre-detection phase, the PPU collects policy information from trustful PAs to generate policy templates. The policy templates are used for generation of substitution policies during the CAPI detection phase.

Regarding the selection of a policy template, the PPU defines two control factors: a similarity value threshold, and the maximum number of selected policy templates. A similarity value is the similarity of attributes of the policy owner of the policy template to that of the unknown policies. 
Service similarity value $=P r_{1} E v_{1}+P r_{2} E v_{2}+P r_{3} E v_{3}+\ldots+P r_{n} E v_{n}$

where $\operatorname{Pr}$ : priority value; Ev: distance of evaluation element values of the policy template to untrustful PA.

A lower service similarity value ( $S S$ value) means higher similarity of policy owners in terms of the evaluation elements. A PPU would define a similarity value threshold and a maximum number of substitution policies for generation of substitution policies in the CAPI detection phase. A policy template would be selected if its similarity value is smaller than the similarity value threshold.

In the CAPI pre-detection phase, after generating policy templates, the PPU searches for the dynamically optimal Priority values. The PPU uses policy similarity value for the calculation. The policy similarity value ( $P S$ value) measures the similarity of two sets of policies:

$$
\text { Policy similarity value }=\frac{\text { Number of matched policies }}{\text { Total number of policies of target policy owner }}
$$

The optimal Priority values result into the highest correlation of $S S$ value and $P S$ value, i.e.: the lower the $S S$ value is, the higher the $P S$ value is. The searching method may be a complete search or a genetic algorithm [7. The evaluation function of the searching can be the weighed $P S$ value of a number of policy template pairs with lowest $S S$ values.

After receiving an authorization request which requires CAPI (i.e., some of the involved PAs are keeping their policy information private), the PPU will enter the Detection phase. To substitute policies for an untrustful PA, the PPU generates substitution policies by selecting from the policy templates. The PPU would then use the substitution policies with the policies from trustful PAs to perform conflict detection. During conflict detection, when a conflict occurs, the corresponding substitution policy would be added into a "Conflict Policy Set". The "Conflict Policy Set" will be used in the CAPI post-detection phase.

After generating the "Conflict Policy Set", the PPU will enter the Postdetection phase. The Conflict Policy Set stores the substitution policies which result into conflicts during the CAPI detection phase. The untrustful PA needs to traverse the policies related to target service to see if any of them is a subset of a policy in the Conflict Policy Set.

For a policy (Policy) untrust in the untrustful PA compared to a policy (Policy) $)_{\text {conflict }}$ in the Conflict Policy Set, (Policy) $)_{\text {original }}$ and (Policy) $)_{\text {conflict }}$ are considered to be matched if the following three criteria are satisfied:

1. (Condition $)_{\text {conflict }}$ is a subset of (Condition) $)_{\text {untrust }}$

2. (Action $)_{\text {conflict }}$ is a subset of (Action) $)_{\text {untrust }}$

3. (Identity) $)_{\text {conflict }}$ is a subset of (Identity) untrust

The PA sends the checking result to the PPU to state the number of "certified conflict policies". The PPU finally makes an authorization decision according to the result. The decision can be based on whether the number of matched conflict policies is within a threshold value which depends on the Grid environment. 


\section{Performance of CAPI}

Since the detection results of CAPI are obtained through estimation, we would like to look into the accuracy of CAPI regarding several different factors. We have done simulation of CAPI based on the following environmental factors:

1. "Correlation of service similarity and policy similarity" (CoSP): It is a real value between 0 and $1 . \operatorname{CoSP}$ represents the likeliness of the statement that "the more similar the two services are (in terms of evaluation element set), the more similar their policy sets are". It is an objective factor to the Grid environment. CoSP can be expressed in the following expression: $\operatorname{CoSP}=\frac{d \text { (Policy similarity })}{d(\text { Service similarity })}$

2. "Maximum number of substitution policies" and "Similarity value threshold": They are used in the selection of substitution policies. They are configurable by the PPU. The similarity value threshold is represented by a percentage value to the range of service evaluation values.

3. "Occurrence rate of opposite policy": It represents the occurrence probability of an opposite policy in the Grid environment. An opposite policy is a policy in conflict to the target policy. This is also an objective factor to the Grid environment.

To evaluate the accuracy of CAPI, we deploy three performance indexes: "Average $P S$ " measures the accuracy of CAPI regarding conflict detection results.

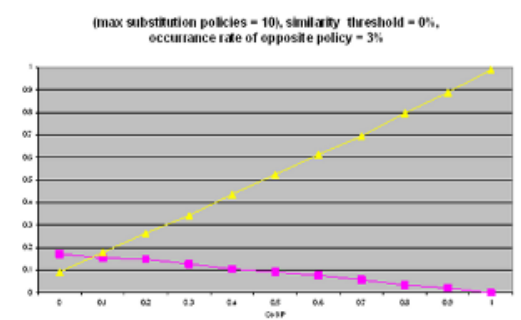

(a)

(imax substitution pelidies $=30 \mathrm{k}$, similarity threstoold $=0 \mathrm{~s}$, eccuriance rate of opposite pelicy $=3$ ?

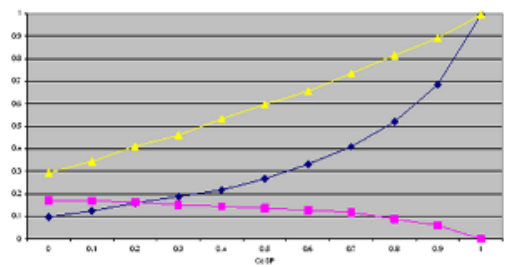

(c)

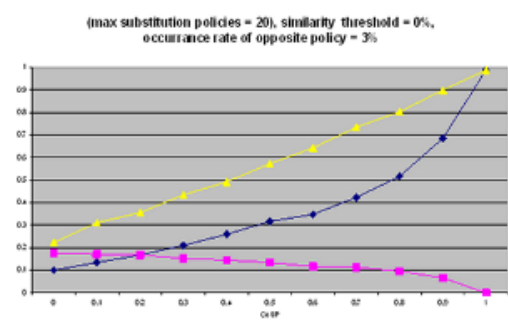

(b)

(max substitution polidies $=40)$, similarihy threshold $=0$, occierrace rate of opposite policy $=3$.

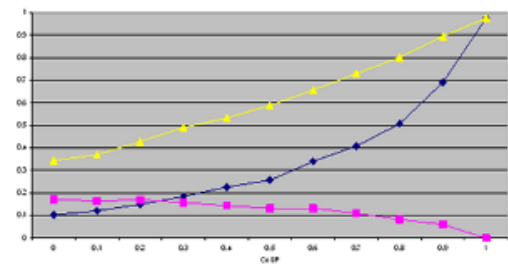

(d)

-Average positive match - - Average negative match $\quad$ Average PS

Fig. 2. Simulation results of different maximum number of substitution policies: (a)10; (b) 20; (c)30; (d)40 
max sultritution policies -20 , (similarity flueshodd $-0<1$.

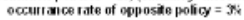

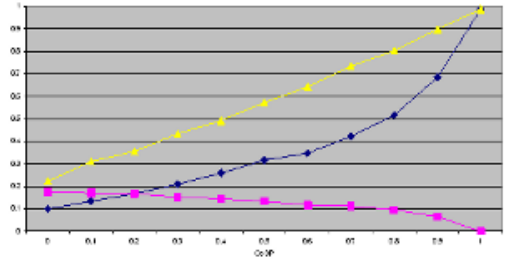

(a)

max substitution policies $=20$, (simbintity threshodd $=2 \times 1$, occurrance rate of opposate pollcy - 3 .

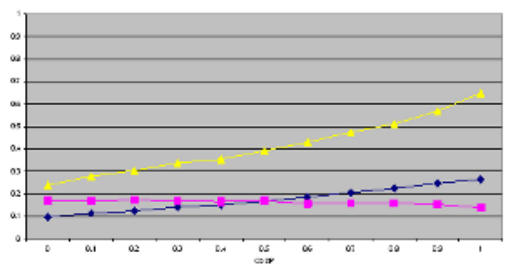

(c) $\max$ substitution policies -20 , \{similatity theshold -1 s, nocuirrance rats of opposite policy $=3$

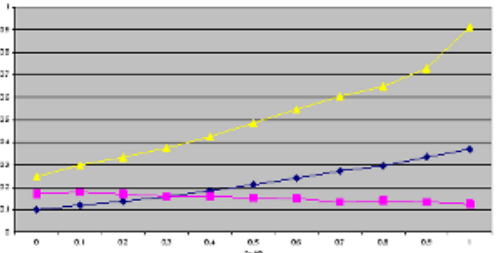

(b)

inax substitution pendes $=20$, (similaritity threstrold $=3 \mathrm{~h}$, occurrance rate of opposine pollcy - 3

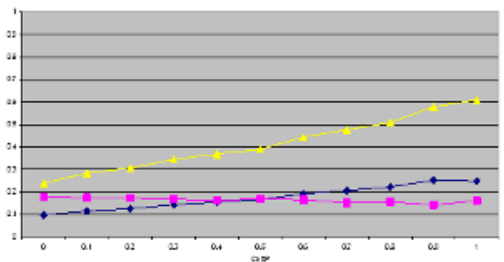

(d)

- Average positive match - -Average negative match - Average PS

Fig. 3. Simulation results of different similarity value threshold: (a)0\%; (b)1\%; (c)2\%; (d) $3 \%$

"Average $P M$ " and "Average $N M$ " measure the quality of generated substitution policies.

1. $P S=\frac{\text { Number of matched policies }}{\text { Total nuber }}$

2. $P M=\frac{\text { Number of matched policies }}{\text { Total number of substitution policies }}$

3. $N M=\frac{\text { Number of opposite policies }}{\text { Total number of substitution policies }}$

From a single diagram in Figure 2 (e.g., Figure 2b) we can see the effect of "Correlation of service similarity and policy similarity" $(C o S P)$ to the accuracy. When $C o S P$ increases:

Average $P S$ increases; Average positive match ( $P M$ ) increases; Average negative match $(N M)$ decreases.

Obviously, $\operatorname{CoSP}$ is a significant factor to the accuracy of CAPI. The higher the $\operatorname{CoSP}$ is, the higher the accuracy of CAPI is.

Figure 2illustrates the effect of "maximum number of substitution policies" to the accuracy of CAPI. Number of policies for each service is 10 . The "maximum number of substitution policies" in Figure 2 ranges from 10 to 40.

When "maximum number of substitution policies" increases:

Average PS increases; Average positive match ( $P M$ ) does not change; Average negative match $(N M)$ does not change significantly.

We can see that a larger "maximum number of substitution policies" can slightly increase the accuracy of the conflict detection result. 
max substitution policies $=20$, similarity threshold $=0 \%$ (occurrance rate of opposite policy $=2 \%$ )

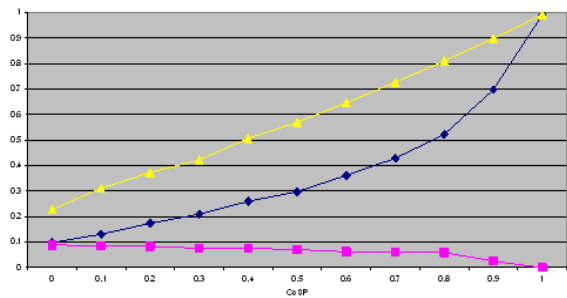

(a)

max substitution policies $=\mathbf{2 0}$, similarity threshold $=0 \%$, (occurrance rate of opposite policy $=4 \%$ )

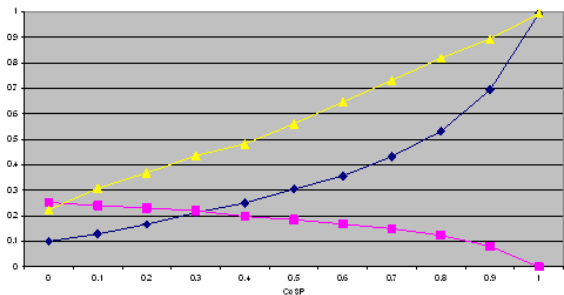

(c) max substitution policies $=20$, similarity threshold $=0 \%$

(occurrance rate of opposite policy $=3 \%$ )

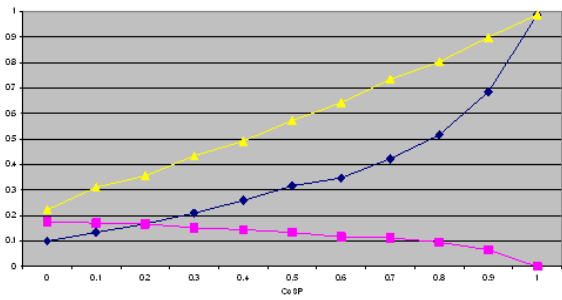

(b)

max substitution policies $=20$, similarity threshold $=0 \%$, (occurrance rate of opposite policy $=5 \%$ )

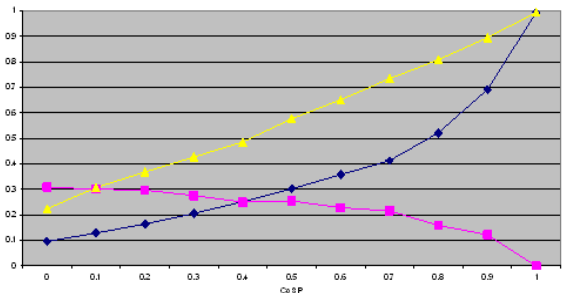

(d)

Average positive match - - Average negative match - Average PS

Fig. 4. Simulation results of different occurrence rate of opposite policy: (a) $2 \%$; (b) $3 \%$; (c) $4 \%$; (d) $5 \%$

Figure 3 illustrates the effect of "similarity value threshold" to the accuracy of CAPI. The "similarity value threshold" in Figure 3 ranges from $0 \%$ to $3 \%$.

When "similarity value threshold" increases:

Average PS decreases; Average positive match ( $P M$ ) decreases; Average negative match $(N M)$ increases.

We can see that the "similarity value threshold" is a significant factor to the accuracy of CAPI. The higher the "similarity value threshold" is, the lower the accuracy of CAPI is.

According to Figure 4, a higher "occurrence rate of opposite policy" results in a higher average negative match $(N M)$ but does not significantly affect average $P S$ and average positive match $(P M)$. Therefore the "occurrence rate of opposite policy" affects the error rate of conflict detection result in CAPI.

A PPU can learn the values of the environment factors during the predetection phase of CAPI. By knowing the environment factors, the PPU can set the threshold value for decision making in the post-detection phase. The PPU makes service authorization decision according to: number of "certified conflict policies" (CCP), negative match $(N M)$ value, positive match $(P M)$ value, and number of substitution policies. Positive authorization would be made if:

$$
\text { Number of } \mathrm{CCP} \leq(1-P M) \times \text { (Number of substitution policies) }
$$


This is because the value $(1-P M)$ represents the error rate of CAPI. Here we assume that the value $(1-P M)$ is larger than the $N M$ value; otherwise, we use $N M$ to replace $(1-P M)$ instead.

\section{Conclusions}

In this paper, we present the Conflict Analysis with Partial Information (CAPI) mechanism. In an open collaboration of VOs, some VOs may want to keep their policy information private. The CAPI mechanism can manipulate service authorization requests when the requests may involve private or unknown policies. CAPI estimates the policies in the unknown policy set, generates substitutions for unknown policies, finds out the ones which can cause conflict, then sends the set of conditions to the untrustful VO for its checking. Simulation results show that CAPI is more suitable to environments where there is a high correlation between the similarity of policy owners' properties and the similarity of their policy sets. Policy decision point can learn the values of environment factors to improve the effectiveness of CAPI.

\section{Acknowledgments}

The work described in this paper was partially supported by a grant from the Research Grants Council of the Hong Kong Special Administrative Region (Project no. CUHK4220/04E).

\section{References}

1. B.E. Carpenter, and P. A. Janson. Abstract Interdomain Security Assertions: A Basis for extra-grid virtual organizations, IBM Systems Journal, Vol. 43, No. 4, 2004, pp. 689-701.

2. Gary N. Stone, Bert Lundy, and Geoffery G. Xie, U.S Department of Defense. Network Policy Languages: A Survey and a New Approach, in IEEE Network, Jan/Feb 2001.

3. J. Strassner and E. Ellesson. Terminology for Describing Network Policy and Services, Internet draft draft-strasner-policy-terms-01.txt, 1998.

4. Dinesh Verma, Sambit Sahu, Seraphin Calo, Manid Beigi, and Isabella Chang: A Policy Service for GRID Computing, M. Parashar(Ed.): GRID 2002, LNCS 2536, pp. 243-255.

5. Von Welch, Frank SiebenSet, Ian Foster, John Bresnahan, Karl Czajkowski, Jarek Gawor, Carl Kesselman, Sam Meder, Laura Pearlman, and Steven Tuecke. Security for Grid Services, in Proceedings of the 12th IEEE International Symposium on High Performance Distributed Computing (HPDC'03).

6. Chiu-Man Yu and Kam-Wing Ng. Dynamic Policy Management Framework for Partial Policy Information, Advances in Grid Computing - EGC 2005. European Grid Conference, Amsterdam, The Netherlands, February 14-16, 2005, Revised Selected Papers, Lecture Notes in Computer Science, vol. 3470, pages 578-588, Springer, June 2005.

7. M. Srinivas and L.M. Patnaik. Genetic Algorithms: A Survey, in IEEE Computer, vol. 27, Issue 6, June 1994, pp. 17-26. 